\title{
Diagnóstico e monitoramento da situação nutricional da população idosa em município da região metropolitana de Curitiba (PR)
}

\author{
Diagnosis and monitoring of the nutritional \\ condition of the elderly in a municipality in the metropolitan \\ region of Curitiba, state of Paraná (PR), Brazil
}

Thais Carolina BASSLER'

Doris Lucia Martini LEI²

\section{RE S U M O}

\section{Objetivo}

O aumento da população idosa em países desenvolvidos, como naqueles em desenvolvimento, desperta interesse e preocupação com o planejamento de políticas públicas voltadas para a atenção nutricional do idoso. O objetivo desta pesquisa foi descrever o perfil nutricional de idosos voluntários pertencentes a grupos paroquiais, para propor o monitoramento da situação nutricional da população idosa no município de Pinhais, na Região Metropolitana de Curitiba (PR).

\section{Métodos}

A população incluída na pesquisa foi de 209 idosos voluntários, pertencentes aos grupos paroquiais de adultos e idosos do município. O diagnóstico do estado nutricional dos idosos foi realizado por meio de medidas antropométricas, com o cálculo do Índice de Massa Corporal, utilizando como critérios diagnósticos os pontos de corte recomendados pelo Ministério da Saúde. Dados sócio-demográficos, como grupo etário, sexo, grau de escolaridade, morbidades, tipo de serviço de saúde utilizado pela população idosa, também foram coletados no período de agosto a novembro de 2003. O Programa Epi Info, versão 6.04, foi utilizado para as análises estatísticas.

\section{Resultados}

Os resultados encontrados mostraram a ocorrência de importantes prevalências de inadequação do estado nutricional na população idosa do Município de Pinhais, onde 57,4\% dos idosos apresentaram sobrepeso e $9,6 \%$ baixo peso.

\footnotetext{
${ }^{1}$ Universidade de Cuiabá, Faculdade de Nutrição. Av. Beira Rio, 3100, Jd. Europa, 78015-480, Cuiabá, MT, Brasil Correspondencia para/Correspondence to: T.C. BASSLER. E-mail: <thacarol@hotmail.com>.

2 Instituto de Saúde Coletiva, Departamento de Nutrição. São Paulo, SP, Brasil.
} 
312 | T.C. BASSLER \& D.L.M. LEI

\section{Conclusão}

Os resultados indicam a necessidade de criar um instrumento de monitoramento da situação nutricional para a população idosa do município, sugerindo o Sistema de Vigilância Alimentar e Nutricional para este grupo etário.

Termos de indexação: Avaliação nutricional. Idoso. Vigilância nutricional.

\section{A B S T R A C T}

\section{Objective}

The increasing elderly population in developed and developing countries arouses interest and concern regarding the planning of public policies which deal with the nutritional care of the elderly. The objective of this research was to describe the nutritional profile of senior volunteers from parochial groups in order to suggest the monitoring of the nutritional situation of the elderly in the municipality of Pinhais, in the metropolitan region of Curitiba, state of Paraná.

\section{Methods}

The population encompassed in the survey consisted of 209 elderly volunteers from the parochial groups of adults and seniors of that city. The diagnosis of the nutritional status of the elderly was done by taking anthropometrical measurements and calculating the Body Mass Index, using the cut-off points recommended by the Ministry of Health as diagnostic criteria. Sociodemographic data, such as age, gender, formal education level, morbidities and type of healthcare service used were also collected from August to November 2003. The Epi Info program, version 6.04, was used to perform the statistical analyses.

\section{Results}

The results showed that important prevalences of inadequate nutritional status were found among the elderly of the city of Pinhais, where $57.4 \%$ of them were overweight and $9.6 \%$ were underweight.

\section{Conclusion}

The results indicated the need to develop an instrument to monitor the nutritional status of the elderly in this city and the Food and Nutritional Surveillance System is proposed for this age group.

Indexing terms: Nutrition assessment. Aged. Nutritional surveillance.

\section{N T R O D U ÇÃ O}

A expectativa de vida tem aumentado no mundo inteiro e, associada à queda dos coeficientes de fecundidade e de mortalidade, tem conduzido ao envelhecimento populacional. Esse fenômeno é característico de países desenvolvidos e também, de modo mais crescente, nos países em desenvolvimento, como é o caso do Brasil, onde esse é o grupo que mais cresce ${ }^{1}$.

O crescente aumento da população idosa mundial tem gerado, para os órgãos governamentais e para a sociedade, o desafio de mudanças médico-sociais próprias do envelhecimento populacional².

O Brasil vem, rapidamente, substituindo as questões da escassez de alimentos por aquelas ligadas à opulência. A desnutrição, embora ainda relevante, vem diminuindo em todas as idades e em todos os estratos econômicos, enquanto o aumento na prevalência da obesidade entre adultos ocorre em todos os estratos econômicos, com aumento proporcional mais elevado nas famílias de mais baixa renda ${ }^{3}$.

A manutenção de um estado nutricional adequado na pessoa idosa é tarefa árdua, frente às doenças crônicas, à associação do uso de medicamentos, às modificações fisiológicas inerentes à idade que interferem no apetite, no consumo e na absorção de nutrientes, e às questões sociais e econômicas que muito prejudicam a prática para a conquista de uma alimentação saudável ${ }^{4}$. 
No âmbito coletivo, a avaliação do estado nutricional é obtida com base em diagnósticos de grupos populacionais em vários níveis (nacional, estadual, municipal e local). No âmbito local, utilizando-se dados da clientela atendida nos serviços de saúde, ou em dados de base populacional, é possível traçar o perfil da população-alvo. Este diagnóstico permite descrever magnitudes, tendências e analisar fatores determinantes, servindo de subsídios para o planejamento e para a tomada de decisões dos gestores da saúde nos segmentos municipal, estadual ou nacional ${ }^{5}$.

Entre as várias formas de avaliação do estado nutricional, em estudos clínicos e principalmente em estudos populacionais, as medidas antropométricas são as mais utilizadas. Este método produz informações básicas das variações físicas e na composição corporal global; é aplicável em todos os ciclos de vida e permite a classificação de indivíduos/grupos em graus de nutrição, além de ser de fácil execução e padronização nos serviços de saúde 6 .

A partir das medidas de peso e estatura, pode-se calcular o Índice de Massa Corporal (IMC), que é habitualmente utilizado na população adulta, devido à grande disponibilidade de dados de massa corporal e estatura, além de sua relação com a morbimortalidade. Esses fatores justificam a utilização do IMC como indicador do estado nutricional em estudos epidemiológicos em associação (ou não) com outras medidas antropométricas ${ }^{7}$.

A coleta contínua dos dados antropométricos, assim como o processamento e a análise dos dados de uma população, possibilita um diagnóstico atualizado da situação nutricional, suas tendências temporais, e também, dos fatores de sua determinação, o que é atribuição do Sistema de Vigilância Alimentar e Nutricional (SISVAN) ${ }^{8}$.

No monitoramento da situação alimentar e nutricional, o SISVAN serve de eixo para o trabalho empreendido na rede de serviços, de forma especial na atenção básica de saúde, inclusive considerando o compromisso de sua univer- salização. Busca-se, também no âmbito da rede de serviços, incorporar às rotinas de atendimento o monitoramento do estado nutricional de cada usuário, visando à detecção da situação de risco e à prescrição de ações que possibilitem a prevenção de seus efeitos e a garantia da reversão ao quadro de normalidade ${ }^{8}$.

A questão dos idosos emerge no âmbito da investigação e do planejamento de políticas públicas voltadas para este grupo etário. Portanto, existe interesse em incorporar às rotinas de atendimento, pela rede de serviços de atenção básica de saúde, o monitoramento do estado nutricional do idoso. O objetivo desta pesquisa foi descrever o perfil nutricional de idosos voluntários pertencentes a grupos paroquiais, para propor o monitoramento da situação nutricional da população idosa no município de Pinhais, na Região Metropolitana de Curitiba (PR).

\section{M É T O D O S}

Foi realizado um estudo transversal, descritivo, no município de Pinhais, Região Metropolitana de Curitiba (PR).

Em parceria com a Pontifícia Universidade Católica do Paraná (PUC/PR), como atividade de extensão universitária, a Prefeitura vem desenvolvendo um trabalho voltado à Educação em Saúde, desde fevereiro de 2002, com 14 grupos de adultos e idosos voluntários que estão vinculados às Paróquias do município, localizadas em diversos bairros, fornecendo a infraestrutura necessária para a realização dos encontros, como transporte e alimentação. Os grupos foram agendados de forma que cada grupo comparecesse em duas reuniões bimestrais (agosto/setembro) e (outubro/novembro) durante o segundo semestre de 2003, em um único local (Paróquia Central), possibilitando uma estratégia de campo para o presente estudo.

A população do estudo foi composta por voluntários incluídos na pesquisa que constituíram um grupo especial, pois integravam os grupos de 
idosos que participavam das atividades sócioculturais desenvolvidas nas paróquias. Medidas antropométricas e dados sócio-demográficos foram coletados junto aos idosos voluntários, pertencentes aos grupos das 14 paróquias do município de Pinhais (PR), nas dependências da Paróquia Central.

Foram investigados 301 indivíduos voluntários, de ambos os sexos, sendo excluídos 86 que apresentaram idade inferior a 60 anos e 1 indivíduo que não soube responder a idade. Dos 214 idosos, 5 (2,3\%) apresentaram algum tipo de limitação física para participar da coleta de medidas antropométricas (1 idoso em cadeira de rodas, 2 que utilizavam muletas e 2 com problemas graves de coluna), que foram, então, excluídos da pesquisa, totalizando uma população de 209 idosos. Participaram do estudo 209 idosos com idade de 60 anos ou mais. Em 2 casos, as pessoas não souberam informar o seu grau de escolaridade.

Os idosos estavam cientes de que a participação era voluntária e de não haver nenhuma conseqüência pela sua não participação.

Os dados sócio-demográficos foram coletados por meio de entrevistas e anotados em formulário específico. As variáveis analisadas foram: faixa etária, sexo, grau de escolaridade, morbidades como diabetes mellitus, hipertensão arterial, asma e bronquite, obstipação, anemia nutricional, entre outras referidas pelos indivíduos e sobre a utilização dos serviços de saúde (público ou privado).

A avaliação do estado nutricional foi realizada por meio de medidas antropométricas de peso $(\mathrm{kg})$ e estatura $(\mathrm{cm})$. O peso foi coletado uma vez por um único examinador, enquanto a estatura foi coletada duas vezes, por dois examinadores distintos.

Os idosos foram pesados em pé, descalços, com o mínimo de vestuário (sem casacos ou malhas), utilizando-se balança eletrônica digital portátil, da marca SOEHNLE, com capacidade de $150 \mathrm{~kg}$ e sensibilidade de $100 \mathrm{~g}$.
A estatura foi medida segundo as técnicas de Frisancho ${ }^{9}$, utilizando-se um estadiômetro portátil (trena de metal acondicionada em um estojo de $15 \times 15 \mathrm{~cm}$ ), de 2 metros e graduado em décimos de $\mathrm{cm}$, comercializado pela Dexter. No local onde foram coletadas as medidas antropométricas, houve a necessidade de utilizar um banco de madeira $(10 \mathrm{~cm}$ de altura $\times 40 \mathrm{~cm}$ de largura e profundidade), confeccionado para eliminar o inconveniente da presença de rodapés na sala da coleta dos dados, de maneira a formar um ângulo reto.

A avaliação nutricional dos idosos foi realizada a partir do IMC $\left(\mathrm{kg} / \mathrm{m}^{2}\right)$, e utilizou como critério de classificação os pontos de corte proposto pelo Nutrition Screening Initiative ${ }^{10}$ e recomendado pelo Ministério da Saúde ${ }^{11}$ : baixo peso: $\leq 22 \mathrm{~kg} / \mathrm{m}^{2}$; eutrófico: $22,1-26,9 \mathrm{~kg} / \mathrm{m}^{2}$ e sobrepeso: $\geq 27 \mathrm{~kg} / \mathrm{m}^{2}$.

Após identificar o estado nutricional dos idosos participantes, os mesmos receberam orientações nutricionais individuais conforme seu IMC (baixo peso, eutrófico e sobrepeso), além de orientações sobre alimentação, a partir de palestras ministradas pelos estagiários durante o semestre.

Os dados sócio-demográficos e as medidas antropométricas foram coletados por 5 estagiários do Curso de Nutrição da Faculdade Integrada Espírita (FIES), que foram treinados e supervisionados pela autora do trabalho.

O Programa Epi Info, versão 6.04², foi utilizado, tendo sido feita dupla digitação para garantir a consistência dos dados. Foi calculado o teste $\chi^{2}$ para verificar a tendência para sobrepeso em função da idade e a correlação do estado nutricional dos idosos segundo o sexo.

Os participantes assinaram um termo de consentimento para a autorização da utilização dos dados de forma sigilosa, com a finalidade de pesquisa científica. O Comitê de Ética em Pesquisa do Instituto de Saúde (CEPIS) aprovou a realização do estudo, sob o número do protocolo 012/03. 


\section{RESULTADOS}

A faixa etária entre 65 e 70 anos foi predominante em relação às demais. O número de idosos abaixo de 70 anos (131) representou 62,6\% deles (Tabela 1). Do total de 209 idosos incluídos na pesquisa, $84,7 \%$ eram do sexo feminino e $15,3 \%$ do sexo masculino.

A pesquisa sobre o grau de escolaridade mostrou que $32,3 \%$ eram analfabetos, pois não sabiam ler/escrever; $27,0 \%$ não completaram a $4^{a}$ série do ensino fundamental (antigo primário) e apenas $13,1 \%$ dos idosos tinham escolaridade igual ou superior a 8 anos de estudo (pelo menos o primeiro grau completo). Observou-se que cerca de $60,0 \%$ dos idosos apresentaram escolaridade inferior a 4 anos de estudo.

Entre as referidas, as três morbidades mais citadas pelos idosos foram a hipertensão arterial, com 59,8\% (116 indivíduos), seguida de problemas de colesterol elevado, com $20,6 \%$, e de diabetes mellitus, com 15,5\% dos entrevistados.

A Tabela 2 expõe as respostas dos idosos a respeito de morbidades, podendo o entrevistado ter referido uma ou mais morbidades $(n=194)$. Do total, 15 indivíduos não referiram nenhum tipo de morbidade.

Dos 116 idosos hipertensos, 80\% só referiram ser portadores dessa doença ( $n=93$ ) e 17,0\% referiram hipertensão arterial mais diabetes mellitus $(n=20)$. Em relação ao colesterol $(n=40)$, $87,5 \%$ dos idosos relataram somente aumento do colesterol $(n=35)$, sendo que $7,5 \%(n=3)$ mencionaram hipertensão arterial mais colesterol

Tabela 1. Distribuição dos idosos segundo sexo e faixa etária. Pinhais (PR), 2003.

\begin{tabular}{|c|c|c|c|c|c|c|}
\hline \multirow{3}{*}{$\begin{array}{l}\text { Faixa etária } \\
\text { (anos) }\end{array}$} & \multicolumn{4}{|c|}{ Sexo } & \multirow{2}{*}{\multicolumn{2}{|c|}{ Total }} \\
\hline & \multicolumn{2}{|c|}{ Masculino } & \multicolumn{2}{|c|}{ Feminino } & & \\
\hline & $n$ & $\%$ & $n$ & $\%$ & $n$ & $\%$ \\
\hline $60 \vdash 65$ & 5 & 8,8 & 52 & 91,2 & 57 & 27,3 \\
\hline $65 \vdash 70$ & 13 & 17,6 & 61 & 82,4 & 74 & 35,3 \\
\hline $70 \vdash 75$ & 7 & 17,9 & 32 & 82,1 & 39 & 18,7 \\
\hline 75 e mais & 7 & 17,9 & 32 & 82,1 & 39 & 18,7 \\
\hline Total & 32 & 15,3 & 177 & 84,7 & 209 & 100,0 \\
\hline
\end{tabular}

Tabela 2. Distribuição de morbidades referidas por idosos. Pinhais (PR), 2003.

\begin{tabular}{lcc}
\hline Tipos de morbidade ${ }^{a}(\mathrm{n}=194)$ & $\mathrm{n}$ & $\mathrm{n}$ \\
\hline Doenças crônicas & 116 & 59,8 \\
Hipertensão arterial & 40 & 20,6 \\
Hipercolesterolemia & 30 & 15,5 \\
Diabetes mellitus & 29 & 14,9 \\
Osteoartrite/reumatismo & 4 & 2,1 \\
Osteoporose & & \\
Inadequação dietética & 27 & 13,9 \\
Obstipação & 9 & 4,6 \\
Anemia nutricional & & \\
Condições gastrointestinais crônicas & 15 & 7,7 \\
Úlcera/Hérnia/Gastrite & & \\
Condições respiratórias & 12 & 6,2 \\
Asma e bronquite & & \\
Problemas psicossociais & 7 & 2,6 \\
Depressão & & \\
Outros & 5 & 2,6 \\
Problemas circulatórios & 4 & \\
Labirintite & & \\
\hline a11 perdas sem informação e 4 não mencionaram nenhuma morbidade.
\end{tabular}

elevado. Quanto ao diabetes mellitus, dos 30 indivíduos, 67,0\% referiram tal morbidade associada com hipertensão arterial $(n=20)$ e $26,0 \%$ apresentaram diabetes mellitus $(n=8)$ isolada.

Outras morbidades referidas foram, em ordem decrescente: osteoartrite/reumatismo (14,9\%), obstipação (13,9\%), úlcera/hérnia/gastrite $(7,7 \%)$, asma e bronquite $(6,2 \%)$, anemia nutricional $(4,6 \%)$ e depressão $(3,6 \%)$.

Da totalidade dos idosos, 73,5\% relataram procurar por uma das Unidades Básicas de Saúde do Município de Pinhais quando precisam de um atendimento à saúde, enquanto $26,5 \%$ são atendidos em consultórios particulares ou por convênios médicos.

Em relação à avaliação nutricional dos idosos, segundo o índice de massa corporal (IMC), o baixo peso $\left(\mathrm{IMC} \leq 22 \mathrm{~kg} / \mathrm{m}^{2}\right)$ foi encontrado em $9,6 \%$ de idosos. Porém, o diagnóstico da maioria, $57,4 \%$, foi de sobrepeso (IMC $\left.\geq 27 \mathrm{~kg} / \mathrm{m}^{2}\right)$, (Tabela 3).

A proporção de baixo peso foi maior no sexo masculino (12,5\%); as mulheres apresen- 
taram maior freqüência de sobrepeso $(60,5 \%$, $p=0,03)$. Encontraram-se valores médios de IMC de $25,76 \mathrm{~kg} / \mathrm{m}^{2}$ para o sexo masculino e $28,31 \mathrm{~kg} / \mathrm{m}^{2}$ para o sexo feminino (Tabela 4).

Os idosos que referiram morbidades crônicas, tais como hipertensão arterial sistêmica (HAS), hipercolesterolemia e diabetes mellitus, apresentaram um aumento do percentual de sobrepeso quando da associação entre duas dessas

Tabela 3. Distribuição percentual (\%) do estado nutricional de idosos, segundo a faixa etária. Pinhais (PR), 2003.

\begin{tabular}{lcccc}
\hline \multirow{2}{*}{$\begin{array}{l}\text { Faixa etária } \\
\text { (anos) }\end{array}$} & $\mathrm{n}$ & \multicolumn{3}{c}{ Estado nutricional (\%) } \\
\cline { 3 - 5 } & & Baixo peso & Eutrófico & Sobrepeso \\
\hline $60 \vdash 65$ & 57 & 7,0 & 28,1 & 64,9 \\
$65 \vdash 70$ & 74 & 14,9 & 28,4 & 56,7 \\
$70 \vdash 75$ & 39 & 2,6 & 35,9 & 61,5 \\
75 e mais & 39 & 10,3 & 46,1 & 43,6 \\
\hline Total & 209 & 9,6 & 33,0 & 57,4 \\
\hline
\end{tabular}

$\chi^{2}$ (teste qui-quadrado) de tendência $=1,35 ; p=0,509$.

Tabela 4. Distribuição percentual (\%) do estado nutricional de idosos segundo o sexo. Pinhais (PR), 2003.

\begin{tabular}{lcccc}
\hline \multirow{2}{*}{ Sexo } & \multirow{2}{*}{$\mathrm{n}$} & \multicolumn{3}{c}{ Estado nutricional (\%) } \\
\cline { 3 - 5 } & & Baixo Peso & Eutrófico & Sobrepeso \\
\hline Masculino & 32 & 12,5 & 46,9 & 40,6 \\
Feminino & 177 & 9,0 & 30,5 & 60,5 \\
\hline Total & 209 & 9,6 & 33,0 & 57,4 \\
\hline
\end{tabular}

$\chi^{2}$ : (teste qui-quadrado) $=4,36 ; \quad p=0,03$.

Tabela 5. Distribuição (\%) do estado nutricional de idosos, segundo as morbidades mais referidas. Pinhais (PR), 2003.

\begin{tabular}{lcccc}
\hline \multirow{2}{*}{ Morbidades } & $\mathrm{n}$ & \multicolumn{3}{c}{ Estado nutricional (\%) } \\
\cline { 3 - 5 } & & Baixo Peso & Eutrófico & Sobrepeso \\
\hline Diabetes mellitus & 8 & 25,0 & 37,5 & 37,5 \\
Colesterol elevado & 35 & 11,4 & 31,4 & 57,2 \\
HAS em associação & 93 & 10,7 & 30,1 & 59,2 \\
Diabetes mellitus e HAS & 20 & - & 35,0 & 65,0 \\
HAS e colesterol & 3 & - & 33,0 & 66,7 \\
Diabetes mellitus e & & & & \\
Colesterol & 2 & - & - & 100,0 \\
\hline
\end{tabular}

HAS: hipertensão arterial sistêmica. morbidades (Tabela 5). Foi maior a proporção de sobrepeso em idosos que referiram diabetes mellitus e HAS (65,0\%), em comparação aos que apresentaram somente diabetes $(37,5 \%)$.

\section{DIS C U S S Ã O}

Os resultados obtidos deverão ser interpretados levando-se em consideração as características da população estudada, restrita a uma clientela de idosos incluídos no estudo por demanda espontânea. É necessário ressaltar que estes resultados não podem ser extrapolados para todos os idosos do município de Pinhais (PR), pois fizeram parte do estudo apenas indivíduos que já freqüentavam os grupos paroquiais.

O grupo etário entre 60 a 70 anos, que compôs a maioria dos idosos avaliados (62,6\%), quando comparado com o grupo com idade superior a 70 anos, demonstrou uma maior disposição em participar das atividades desenvolvidas nas paróquias.

O predomínio de idosos do sexo feminino $(84,7 \%)$ sugere que as mulheres são mais participativas das atividades sócio-culturais das paróquias do que os homens. Cervato et al. ${ }^{13}$, ao avaliarem uma intervenção educativa entre idosos freqüentadores de Universidades Abertas para a Terceira Idade no município de São Paulo (SP), verificaram o predomínio da população feminina neste trabalho. Segundo resultados encontrados na pesquisa "Saúde e bem-estar no envelhecimento" (SABE) ${ }^{14}$, assim como dos últimos censos e das contagens populacionais, a parcela de mulheres é maior do que a de homens entre a população idosa, uma vez que as mulheres representaram $58,6 \%$ do universo estudado de idosos paulistanos.

A maioria dos entrevistados, cerca de 73,0\%, informou estar vinculada e ser usuária do Sistema Único de Saúde (SUS), quando necessita de algum tipo de atendimento à saúde no município. Este número mostrou a alta demanda 
da população idosa aos serviços públicos de saúde do município. Resultados encontrados por Cesar \& Paschoal, no projeto SABE ${ }^{14}$ do município de São Paulo, demonstraram resultados semelhantes, pois cerca de $60,0 \%$ da população idosa é usuária do SUS.

Em relação à categorização do grau de escolaridade, quase $60,0 \%$ dos idosos de Pinhais referiram até quatro anos de estudo, $27,0 \%$ de 4 a 7 anos e 13,1\% estudaram por 8 anos ou mais. Estes resultados mostram a grande concentração de idosos com baixa escolaridade, muito próximos dos valores encontrados no projeto $\mathrm{SABE}^{14}$, segundo o qual cerca de $78,0 \%$ da população idosa possuíam até 4 anos de escolaridade, 9,0\% freqüentaram a escola por 5 a 8 anos e 13,1\% têm acima de 8 anos de estudo.

A multiplicidade de morbidades em um mesmo idoso é muito freqüente. Para Ramos et al. ${ }^{15}$, apenas $14 \%$ dos idosos disseram-se livres de doenças crônicas, enquanto 15\% deles mencionaram cinco ou mais doenças.

No estudo realizado por Frank et al. ${ }^{16}$, a HAS foi a enfermidade mais citada pelos idosos entrevistados, seguida de diabetes mellitus e de hiperlipidemias. Do total da população estudada, 18\% negaram qualquer tipo de doença, estando, assim, de acordo com os resultados encontrados por este estudo.

Nos idosos que referiram doenças crônicas, observou-se uma tendência no aumento da freqüência de sobrepeso (60\%). Dessa forma, existe grande necessidade de implantar algum tipo de assistência nutricional à população idosa pelo município, devido às possíveis conseqüências relacionadas com o sobrepeso, como as doenças crônicas não-transmissíveis.

De acordo com o mapa de acompanhamento diário para a Vigilância Alimentar e Nutricional, proposto pelo Ministério da Saúde ${ }^{11}$, as morbidades questionadas aos idosos e aos demais usuários são, entre elas: HAS, diabetes não insulino-dependente e doenças cardiovasculares.
Algumas doenças potencializadas pela obesidade assumem importância maior entre os idosos, pois já apresentam freqüências aumentadas com a idade em indivíduos idosos não obesos $^{15}$.

A classificação do estado nutricional de idosos segundo o IMC é, ainda, bastante discutida, havendo divergência, entre vários estudos, quanto aos valores empregados ${ }^{6,17,18}$.

O presente estudo encontrou valores médios de IMC de $25,76 \mathrm{~kg} / \mathrm{m}^{2}$ para o sexo masculino e $28,31 \mathrm{~kg} / \mathrm{m}^{2}$ para o sexo feminino. Quando comparados os valores de IMC dos idosos deste estudo, com dados citados por Coitinho et al. ${ }^{19}$, verificase uma semelhança em relação ao estado nutricional. A prevalência de sobrepeso, encontrada por Coitinho et al. ${ }^{19}$ na população idosa, foi de $30,4 \%$ em homens e 50,2\% em mulheres, predominando esse problema no sexo feminino em diferentes faixas etárias, entre elas as de 65 anos e mais. Recentemente, a Pesquisa de Orçamentos Familiares (POF), realizada em 2002/2003 pela Fundação Instituto Brasileiro de Geografia e Estatísitca $(\mathrm{IBGE})^{20}$, relacionou os hábitos alimentares com os dados antropométricos da população brasileira de 20 anos ou mais de idade. Os dados na população idosa brasileira, estratificada por sexo e faixa etária, apontaram déficit de peso de $8,9 \%$, em homens com 75 anos ou mais de idade; as mulheres de ambas as faixas etárias ( 65 a 74 anos e 75 anos ou mais) não apresentaram déficit de peso ${ }^{20}$. A partir dos 65 anos de idade, tanto para homens quanto para mulheres, observa-se tendência de declínio na prevalência do excesso de peso; para os homens foi de 43,9\% (65 a 74 anos) e de 33,3\% (75 anos ou mais). Para as mulheres os resultados foram de 53,3\% (65 a 74 anos) e de $42,5 \%$ (75 anos ou mais) de excesso de peso. O mesmo ocorre com a obesidade; para homens foi, respectivamente, de $10,2 \%$ e 5,6\%; para mulheres, de $17,1 \%$ e $14,3 \%^{20}$.

Outros estudos mostram valores diferentes de prevalência de sobrepeso e baixo peso na população idosa brasileira. Tavares \& Anjos $^{6}$ 
registraram uma prevalência geral de sobrepeso $\left(\mathrm{IMC}>25 \mathrm{~kg} / \mathrm{m}^{2}\right)$ de $30,4 \%$ em homens e de $50,2 \%$ em mulheres, enquanto a prevalência geral de magreza $\left(\mathrm{IMC}<18,5 \mathrm{~kg} / \mathrm{m}^{2}\right)$ foi de $7,8 \% \mathrm{em}$ homens e de $8,4 \%$ em mulheres. Martins et al. ${ }^{21}$, utilizando os mesmos pontos de corte, verificaram que a prevalência de sobrepeso foi de $34,1 \%$ em homens e de 40,3\% em mulheres e a de baixo peso foi de 7,3\% em homens e de 8,1\% em mulheres, tendo o baixo peso (IMC $18,8-19,9 \mathrm{~kg} / \mathrm{m}^{2}$ ) atingido $19,5 \%$ dos homens e $4,8 \%$ das mulheres.

É importante considerar que o problema da desnutrição continua existindo no Brasil, ainda que, diferentemente da obesidade, afetando contingentes populacionais mais limitados ${ }^{3}$. Problemas nutricionais estão associados ao aumento da morbidade e da mortalidade e com impacto negativo na qualidade de vida entre idosos. Na meia idade (50 a 65 anos) o maior problema nutricional é o sobrepeso, estando associado às doenças crônicas não-transmissíveis. Acima dos 80 anos, magreza e perda de massa magra são os maiores problemas. As doenças associadas com o baixo IMC são tuberculose, enfermidades pulmonares obstrutivas e câncer de pulmão e de estômago 22 .

No entanto, a avaliação nutricional do idoso apresenta limitações também em relação aos dados, e padrões de referência para as medidas antropométricas. Nos países em desenvolvimento, inexistem dados ou padrões para esse grupo etário. Nos países desenvolvidos, os estudos para a construção desses dados e padrões foram realizados a partir de amostras representativas, porém com limite superior de idade ${ }^{9,23}$.

Apesar de a Organização Mundial de Saúde ${ }^{24}$ sugerir, para avaliação do idoso, a utilização dos pontos de corte propostos para o adulto jovem $\left(I M C<18,5 \mathrm{~kg} / \mathrm{m}^{2}=\right.$ magreza e $I M C>30 \mathrm{~kg} / \mathrm{m}^{2}=$ obesidade), supõe-se que valores superiores sejam mais adequados, já que o idoso necessita de uma reserva maior, no sentido de prevenir a desnutrição. Na prática clínica geriátrica, o IMC $<20 \mathrm{~kg} / \mathrm{m}^{2}$ é o ponto de corte adotado para classificar o idoso como apresentando magreza.
Este critério é utilizado para que indivíduos em risco nutricional sejam detectados precocemente ${ }^{25}$.

Segundo Lipschitz ${ }^{26}$, é recomendável que as pessoas acima de 65 anos de idade, apresentem um IMC entre 24 e $29 \mathrm{~kg} / \mathrm{m}^{2}$. Para Stevens ${ }^{27}$, é considerado obeso o indivíduo que apresenta IMC maior ou igual a $30 \mathrm{~kg} / \mathrm{m}^{2}$. É recomendável que os idosos ( $>60$ anos) ${ }^{14}$ apresentem um IMC entre 23 e $28 \mathrm{~kg} / \mathrm{m}^{2}$.

O Ministério da Saúde ${ }^{11}$ recomendou, em última publicação, para os procedimentos de diagnóstico e acompanhamento do estado nutricional de idosos (60 anos e mais) em serviços de saúde, a classificação do IMC seguindo os mesmos pontos de corte do NSI ${ }^{10}$ : baixo peso $I M C \leq 22 \mathrm{~kg} / \mathrm{m}^{2}$; peso normal ou eutrófico de 22 a $27 \mathrm{~kg} / \mathrm{m}^{2}$ e sobrepeso $I M C \geq 27 \mathrm{~kg} / \mathrm{m}^{2}$.

O envelhecimento populacional determina um substancial aumento, tanto nos recursos materiais e humanos necessários aos serviços de saúde do País, como nos seus custos, visto que, em geral, as doenças que acometem essa camada da população necessitam de tratamento por períodos prolongados e intervenções caras com alta tecnologia².

O registro, a análise e o entendimento das transformações do perfil de saúde de uma comunidade evidenciam possíveis estratégias para a melhoria da questão da qualidade de vida, que aponta a necessidade de privilegiar ações voltadas para a prevenção de doenças crônicas, que, em algum momento, atingem os indivíduos. Com esse retardamento do início da doença crônica, o tempo de vida saudável se ampliaria e a necessidade de utilização de serviços de saúde se reduziria ${ }^{28}$.

Este fato vem ao encontro do interesse do gestor de saúde do município, em termos de custo-benefício.

As principais funções que o enfoque nutricional desempenha nesse contexto são: a identificação de indivíduos em risco para desenvolver doenças crônicas não-transmissíveis e a intervenção alimentar para a prevenção e o controle de enfermidades 5 . 
As políticas para melhorar a nutrição conduzem a programas específicos. Quando se trata de elaborar um programa determinado e vigiar seus efeitos, necessitam-se de informações nutricionais específicas. A operacionalização da Vigilância Alimentar e Nutricional (VAN) consiste em como, na prática, serão produzidas e analisadas as informações sobre perfil alimentar e nutricional da população. Isto é, quais estruturas ou rotinas serão criadas ou adaptadas, quais os profissionais e as instituições envolvidas para a produção, o processamento, a análise e a divulgação dessas informações ${ }^{29}$.

Para a operacionalização de um SISVAN, o fundamental é ter um eixo central básico, padronizado entre as instituições que compõem o sistema, e ter flexibilidade suficiente para respeitar as peculiaridades de cada local. Dois aspectos são fundamentais para sua viabilização: assumir a vigilância nutricional como atividade integrante dos Programas de Atenção Integral à Saúde e a inseri-la em rotinas e normas já estabelecidas nos serviços, que sejam desenvolvidas de forma satisfatória ${ }^{29}$.

Apesar de os indivíduos aqui estudados não representarem a população idosa do município, pode-se perceber, pelos resultados encontrados, a importância da VAN. Foi possível diagnosticar a situação nutricional do grupo de idosos, o que permitiria desencadear ações imediatas e planejar políticas públicas a médio e longo prazos, direcionadas à melhoria dessas condições. Nesse contexto, o SISVAN abre caminhos para se tornar parte da atuação básica de saúde, com base nos princípios do SUS, promovendo saúde no plano local.

A Secretaria de Saúde do Município de Pinhais (PR), atualmente, operacionaliza o SISVAN não informatizado, em todas as Unidades Básicas de Saúde (UBS) para crianças de 0 a 59 meses, gestantes e lactantes. Dessa forma, o SISVAN faz parte da rotina dos atendimentos diários nas UBS e possui uma infraestrutura mínima necessária como profissionais, espaço físico e material técnico para coletar, de forma contínua, os dados necessá- rios. Assim, torna-se mais acessível inserir o monitoramento nutricional dos idosos nos serviços de saúde, utilizando os dados produzidos na rotina do Serviço de Saúde, como: identificação do paciente, peso $(\mathrm{kg})$, estatura $(\mathrm{m})$, pressão arterial, temperatura axilar (para subsidiar as consultas), diagnóstico e conduta do profissional de saúde (registro no prontuário para acompanhamento da evolução do paciente).

Para a consolidação do monitoramento nutricional de uma coletividade, vale ressaltar que as UBS do município funcionam como espaços estratégicos para a Vigilância Nutricional, além de outras fontes de dados que poderão ser utilizadas pelos profissionais responsáveis pela VAN, como pesquisas e estudos populacionais, Programa Saúde da Família, Programa Agente Comunitário de Saúde, creches, escolas e outras entidades pertinentes ${ }^{11}$.

O panorama da saúde do idoso no Brasil precisa ser, sem dúvida, melhor definido populacionalmente e em todas as regiões. É imprescindível que se estabeleça uma política de saúde que implique na promoção da qualidade de vida e da autonomia, de modo acessível a todos os estratos sociais ou, pelo menos, que os serviços sejam oferecidos de modo digno aos mais carentes na hierarquia social, para que possam conduzir o seu envelhecimento de maneira saudável e o mais independentemente possível ${ }^{30}$.

Este estudo demonstrou a importância das informações coletadas sobre o estado nutricional de idosos, indicando a necessidade de ações efetivas no controle do agravo à saúde, assim como atuação do município na proteção, prevenção e na recuperação da saúde.

Diante dos resultados apresentados, e por se tratar de um assunto relativamente novo, faz-se necessária a obtenção de novas informações nutricionais, a partir de levantamentos antropométricos populacionais periódicos e da coleta sistemática e contínua de dados provenientes dos serviços de saúde, para que se possam estabelecer práticas de monitoramento nutricional e direcionar intervenções mais adequadas. 


\section{COLABORADORES}

T.C. BASSLER participou da coleta de dados, da tabulação, da discussão dos resultados e da elaboração do artigo. D.LM. LEI participou da elaboração de estratégia experimental da tabulação, da discussão dos resultados e da elaboração do artigo.

\section{REFERÊ N CIAS}

1. Ramos LR, Veras RP, Kalache A. Envelhecimento populacional: uma realidade brasileira. Rev Saúde Pública. 1987; 21(3):211-21.

2. Papaléo Netto M, Ponte JR. Envelhecimento: desafio na transição do século. In: Papaléo Netto M. Gerontologia. São Paulo: Atheneu; 1996. p.3-6.

3. Monteiro CA, Mondini L, Souza ALM, Popkin BM. Da desnutrição para a obesidade: a transição nutricional no Brasil. In: Monteiro CA, organizador. Velhos e novos males da saúde no Brasil: a evolução do país e suas doenças. 2a. ed. São Paulo: Hucitec; 2000. p.247-55.

4. Silva MLT. Geriatria. In: Waitzberg DL. Nutrição oral, enteral e parenteral na prática clínica. 3a. ed. São Paulo: Atheneu; 2000. p.997-1008.

5. Silva DO. SISVAN: instrumento pra o combate aos distúrbios nutricionais na atenção à saúde: o diagnóstico coletivo. Rio de Janeiro: Fiocruz; 2002.

6. Tavares EL, Anjos LA. Perfil antropométrico da população idosa brasileira. Resultados da pesquisa nacional sobre saúde e nutrição. Cad Saúde Pública. 1999; 15(4):759-68.

7. Anjos LA. Índice de massa corporal como indicador do estado nutricional de adultos: revisão da literatura. Rev Saúde Pública. 1992; 26 (6):431-6.

8. Brasil. Ministério da Saúde. Secretaria de Políticas de Saúde. Departamento de Formulação de Políticas de Saúde. Política nacional de alimentação e nutrição. Brasília: Ministério da Saúde; 2000.

9. Frisancho AR. New standards of weight and body composition by frame size and height for assessment of nutritional status of adults and elderly. Am J Clin Nutr. 1984; 40(4):808-19.

10. Incorporating Nutrition Screening and Interventions into Medical Practice. A monograph for phycicians. The Nutrition Screening Initiative. Washington (DC): The American Dietetic Association; 1994.

11. Brasil. Ministério da Saúde. Coordenação Geral da Política de Alimentação e Nutrição. Orientações básicas para a coleta, processamento, análise de dados e informação em serviços de saúde para o sistema de vigilância alimentar e nutricional. Versão Preliminar. Brasília: Ministério da Saúde; 2004.

12. Dean AG, Dean JÁ, Burton AH, Dicker RC. Epi Info, version: 6.04: a word processing database and statistics program for epidemiology on microcomputers. Atlanta: Centers for Disease Control and Prevention; 1997.

13. Cervato AM, Derntl AM, Latorre MRDO, Marucci MFN. Educação nutricional para adultos e idosos: uma experiência positiva em universidade aberta para a terceira idade. Rev Nutr. 2005; 18(1):41-52.

14. César CLG, Paschoal SMP. Uso dos serviços de saúde. In: Lebrão ML, Yeda AOD. SABE - Saúde, bem-estar e envelhecimento - o projeto Sabe no município de São Paulo: uma abordagem inicial. Brasília: Organização Pan-Americana da Saúde; 2003. p.227-37.

15. Ramos LR, Rosa TEC, Oliveira ZM, Medina MCG, Santos FRG. Perfil do idoso em área metropolitana na Região Sudeste do Brasil: resultados de inquérito domiciliar. Rev Saúde Pública. 1993; 27(2): 87-94.

16. Frank AA, Ramos FRM, Machado JS, Souza W, Vidal AT. Perfil nutricional de idosos freqüentadores da associação recreativa de Copacabana, Rio de Janeiro. Nutr Brasil. 2004; 3(2):85-91.

17. Al Snih S, Markides KS, Ray L, Ostir GV, Goodwin JS. Handgrip strength and mortality in older Mexican Americans. J Am Geriatr Soc. 2002; 50(7): 1250-6.

18. Melton JL, Kosla S, Crowson CS, O'Connor MK, O'Fallon M, Riggs L. Epidemiology of sarcopenia. J Am Geriatr Soc. 2000; 48(6):625-30.

19. Coitinho DC, Leão MM, Recine E, Sichieri R. Pesquisa nacional sobre saúde e nutrição: condições nutricionais da população brasileira: adultos e idosos. Brasília: INAN; 1991.

20. Instituto Brasileiro de Geografia e Estatistica. Ministério do Planejamento, Orçamento e Gestão. Análise da disponibilidade domiciliar de alimentos e do estado nutricional no Brasil. POF 2002-2003. Rio de Janeiro; 2004. [acesso em 2005 jan 18]. Disponível em: <http://www.ibge.gov.br>.

21. Martins IS, Velásquez-Meléndez G, Cervato AM. Estado Nutricional de grupamentos sociais da área metropolitana de São Paulo, Brasil. Cad Saúde Pública. 1999; 15(1):71-8.

22. World Health Organization. Physical Status: the use and interpretation of anthropometry. Geneva; 1995. Who Technical Report Séries 854.

23. Chumlea WC, Roche AF, Mukherjee D. Nutritional assessment of the elderly through anthropometry. Ohio: Wright State University School of Medicine; 1987. 
24. World Health Organization. Obesity: Preventing and managing the global epidemic. Geneva; 1998. Report of a WHO Consulation on Obesity.

25. Perissinotto E, Pisent C, Sergi G, Grigoletto F, Enzi $\mathrm{G}$. Anthropometric measurements in the elderly: Age and gender differences. Br J Nutr. 2002; 87(2):177-86.

26. Lipschitz DA. Screening for nutritional status in the elderly. Primary Care. 1994; 21(1):55-67.

27. Stevens J. Impact of age an associations between weight and mortality. Nut Rev. 2000; 58(5): 129-137.

28. Barreto ML, Carmo EH. Mudanças em padrões de morbi-mortalidade: conceitos e métodos. In: Monteiro CA, organizador. Velhos e novos males da saúde no Brasil: a evolução do país e suas doenças. 2a. ed. São Paulo: Hucitec; 2000. p.17-30.

29. Escola Nacional de Saúde Pública. Programa de Apoio à Reforma Sanitária. O sistema de vigilância alimentar e nutricional na rede de saúde: manual para implantação. Rio de Janeiro: ENS; 1993.

30. Lessa I. O adulto brasileiro e as doenças da modernidade. Epidemiologia das doenças crônicas não-transmissíveis. São Paulo: Hucitec Abrasco; 1998.

Recebido em: 2/8/2006

Versão final reapresentada em: 22/5/2007

Aprovado em: 29/2/2008 